\title{
Estrategias familiares de vida y su relación con desnutrición en niños menores de dos años
}

\author{
A raceli Amada Sandoval-Priego, MCS, ${ }^{(1)}$ Hortensia Reyes-Morales, M en $C_{1}{ }_{1}^{(2)}$ \\ Ricardo Pérez-Cuevas, M en C, ${ }^{(2)}$ Rebeca A brego-Blas, MC, ${ }^{(3)}$ Efrén Samuel O rrico-Torres, MC. ${ }^{(4)}$
}

\section{Sandoval-Priego AA, Reyes-Morales H, Pérez-Cuevas R, Abrego-Blas R, Orrico-Torres ES. Estrategias familiares de vida y su relación con desnutrición en niños menores de dos años. Salud Publica Mex 2002;44:41-49. \\ El texto completo en inglés de este artículo está disponible en: http://www.insp.mx/salud/index.html}

\section{Resumen}

Objetivo. Identificar las estrategias familiares de vida asociadas con desnutrición en niños menores de dos años. Material y métodos Estudio de casos y controles efectuado en 1998 en el municipio de Teolocholco, estado de Tlaxcala, México, para estudiar familias con niños de 6 a 23 meses de edad. La muestra estuvo conformada por 105 casos y 210 controles. Las estrategias familiares de vida se agruparon en cinco tipos: fo rmación de la familia, obtención y organización de recursos familiares, colaboración intra o extrafamiliar y preservación de la vida. La desnutrición se identificó de acuerdo con la talla para la edad. Para el análisis de los datos se hizo estimación de razones de momios, con intervalos de confianza al $95 \%$ y se construyeron modelos de regresión logística no condicionada. Resultados Se obtuvieron datos de 605 unidades familiares, 445 controles y 160 casos. El modelo predictivo estuvo integrado por la escolaridad de la madre, hacinamiento, intervalo entre nacimientos, ingreso per capita mensual y el tiempo destinado para actividades de crianza/cuidado de los niños. Conclusiones Las estrategias familiares de vida son determinantes para la ocurrencia desnutrición. Las características de la vida familiar deben considerarse en programas dirigidos a abatir el padecimiento en este grupo. El texto completo en inglés de este artículo está disponible en: http://www.insp.mx/salud/index.html

Palabras clave: desnutrición proteico-energética; familia; estrategias; producción de salud en el hogar; México

\author{
Sandoval-Priego AA, Reyes-Morales H, Pérez-Cuevas R, \\ Abrego-Blas R, Orrico-Torres ES. \\ Family life strategies associated \\ with malnutrition in children aged under two years. \\ Salud Publica Mex 2002;44:41-49. \\ The English version of this paper \\ is available at: http://www.insp.mx/salud/index.html
}

\begin{abstract}
Objective. To identify the role of family life strategies on malnutrition in children aged 6-23 months of age. Material and Methods This case-control study was conducted in 1998 in the municipality of Teolocholco, State of Tlaxcala, Mexico, among families with children aged 6-23 months of age. The sample was conformed by 105 cases and 210 controls. Family life strategies were grouped into five types: family composition, means and distribution of family income, family and social networks, and life preservation strategies. Malnutrition was classified according to height for age. D ata were analyzed using logistic regression to obtain odds ratios and $95 \%$ confidence intervals. Results. D ata were collected from 605 families, for a total of 445 controls and 160 cases. The predictive model included mother's schooling, overcrowding, time elapsed between childbirths, per capita monthly income, and time devoted to child-rearing activities. Conclusions. Family life strategies determine children's nutritional status; understanding the influence of the family on the children's health status is necessary to develop effective programs aimed at improving the nutritional status of children. The English version of this paper is available at: http://www.insp.mx/salud/index.html
\end{abstract}

Key words: protein-energy malnutrition; family; strategies; production of health; Mexico

Este trabajo de investigación fue financiado por El Consejo N acional de Ciencia yTecnología (C onacyt).N úmero de registro:98 M.I.G.29193-m.Coordinación de Investigación Médica del Instituto Mexicano del Seguro Social. N 0 . de registro FP. 0038/422

(1) Hospital General de Subzona C/Unidad de Medicina Familiar N 0.8, Delegación Estatal en Tlaxcala, Instituto Mexicano del Seguro Social, Tlaxcala, México.

(2) Unidad de Investigación Epidemiológica y en Servicios de Salud, Centro Médico N acional Siglo XXI, Instituto Mexicano del Seguro Social, México, D.F., México.

(3) Unidad de Medicina Familiar N 0.51, D elegación Estatal en Tlaxcala, Instituto Mexicano del Seguro Social,Tlaxcala, México.

(4) Jefatura D elegacional de Prestaciones Médicas, D elegación Estatal en Tlaxcala, Instituto Mexicano del Seguro Social,Tlaxcala, México.

Fecha de recibido: 30 de marzo de 2001 • Fecha de aprobado: 28 de agosto de 2001 Solicitud de sobretiros:A raceli A mada Sandoval Priego, 51 N orte N 0. 1011, Col.Aquiles Serdán, 72140 Puebla, Pue. México. correo electrónico: rabasp@yahoo.com 
a desnutrición es uno de los grandes problemas de salud que enfrenta el mundo en la actualidad. Este problema se encuentra vinculado con más de $41 \%$ de las muertes que se presentan anualmente en niños de 6 a 24 meses de edad en los países en desarrollo, ${ }^{1} \mathrm{y}$ que suman aproximadamente 2.3 millones. En los niños sobrevivientes, las secuelas afectan de manera permanente su calidad de vida e implican pérdidas millonarias. ${ }^{2,3}$

En México, la Encuesta Nacional de Nutrición realizada durante 1999 constató una prevalencia de desmedro en niños menores de cinco años de $17.7 \%$, y en niños entre 12 y 23 meses de $21.6 \%$. La frecuencia de este problema disminuyó en forma desigual con respecto a los datos de 1988, ya que en el grupo de menores de cinco años el descenso en términos relativos fue de $-22.3 \%$, mientras que en el grupo entre uno y dos años el descenso fue sólo menor de - $12.1 \%$; lo anterior refleja que el problema permanece prácticamente sin modificaciones en los niños de esta edad. ${ }^{4,5}$

Las principales repercusiones del desmedro en niños menores de dos años de edad son 1) el incremento en la mortalidad, estimado en $20 \%{ }^{6}$ y relacionado con la sinergia que se establece entre la desnutrición aситиlativa y la presencia de procesos infecciosos agudos; ${ }^{7}$ 2) la "discapacidad intelectual" productiva y de desarrollo en los niños sobrevivientes, ${ }^{8}$ que está vinculada con la creatividad, desempeño en el trabajo y la movilidad social, y 3) la afectación de tipo intergeneracional; las madres de talla baja tienen hijos que nacen con bajo peso y talla pequeña, con una elevada probabilidad de tener déficit de talla en etapas posteriores de la vida,

Se han identificado numerosos factores relacionados con la ocurrencia de desnutrición, como ingesta insuficiente calórico proteica, carencias simultáneas de varios micronutrientes (vitamina A, hierro, zinc), ${ }_{1}^{11}$ cambios en los patrones de alimentación que se llevan a cabo durante los primeros dos años de vida, ${ }^{12}$ episodios de enfermedades infecciosas agudas como parasitosis intestinal y algunos padecimientos crónicos, como la enfermedad por Helicobacter pylori. ${ }^{13}$ Otros aspectos han sido las condiciones del entorno, como el nivel socioeconómico, las características de los cuidadores de los niños en las familias ${ }^{14}$ y las inherentes al individuo como el peso al nacer, el género y la edad. ${ }^{15}$ Sin embargo, en la mayoría de los estudios estos factores se comunican de manera aislada y el nivel de análisis con frecuencia carece de un enfoque que explique la desnutrición en una forma integral.

No es posible abordar el tema de la desnutrición sin considerar los factores económicos, sociales y culturales asociados con su ocurrencia. ${ }^{16,17}$ En consecuencia, surge la necesidad de un abordaje integral que permita identificar, a través de dichos factores, los niños con riesgo de ser afectados. La familia parece un punto de partida apropiado como unidad intermedia de análisis para la vinculación entre los distintos factores arriba mencionados. La familia es una institución de la sociedad que tiene un papel mediador entre el contexto macrosocial y el individuo. Para los fines de este trabajo la familia se definió como el grupo de personas que comparten la misma vivienda, gastos para la alimentación y se hallan unidos por lazos de parentesco o afinidad. De acuerdo con las condiciones de existencia que les impone su pertenencia a un determinado ambiente social, las familias desarrollan arreglos domésticos, comportamientos o acciones para satisfacer las necesidades básicas de sobrevivencia. ${ }^{18,19}$

Los procesos de toma de decisiones que repercuten en distintos aspectos de la vida familiar se pueden conceptualizar como estrategias familiares de vida, y éstas consisten en formación de la familia, sus características estructurales, los mecanismos para obtener los recursos necesarios, la distribución de los recursos financieros, la división del trabajo doméstico por edad y género, la realización de acciones de atención a la salud o a la enfermedad y las pautas de interacción con el entorno social. ${ }^{20,21}$ Así, cada familia establece su propio esquema de vida y la selección de un conjunto determinado de estrategias familiares repercute en el estado de salud de sus integrantes, aun cuando las familias compartan un ambiente socioeconómico similar. El niño menor de dos años es uno de los elementos más vulnerables de la familia, y sus condiciones de salud y nutricionales representan un indicador apropiado de la salud de la propia familia.22

El presente trabajo tiene como propósito identificar, en familias con uno o más niños menores de dos años de edad, las estrategias familiares de vida, y trata de establecer la relación potencial entre estas estrategias y la aparición de desnutrición en niños menores de dos años de edad.

\section{Material y métodos}

Se llevó a cabo un estudio de casos y controles durante el segundo semestre de 1998 en el municipio de San Luis Teolocholco, situado en la región sudeste del estado de Tlaxcala, México, área en transición de rural a urbana. Al momento del estudio, el municipio contaba con 16 548 habitantes y aproximadamente 3009 familias. Como unidades de estudio se incluyeron familias con uno o más niños de 6 a 23 meses de edad. El tamaño de muestra se calculó utilizando la fórmula para diseño de casos y controles no pareado con relación caso: control 1:2; nivel a de 0.05 , poder de $80 \%$ y razón de momios 
mínima esperada de 2.5. Con base en estimaciones censales, se consideró a la migración laboral como la variable con menor frecuencia $(17.0 \%)$ de exposición entre los controles. El total de familias requeridas fue de 105 casos y 210 controles.

Las variables independientes fueron las estrategias familiares de vida (EFV) y se agruparon en cinco tipos: a) formación de la familia integrada por las siguientes dimensiones: constitución de la familia, procreación, composición y estructura familiar; b) obtención de los recursos familiares: características de las actividades laborales de los perceptores económicos, ingreso familiar mensual, ingreso familiar per capita y migración laboral; c) organización intrafamiliar de los recursos: división del trabajo doméstico, distribución de recursos financieros, de servicios y tiempo; d) colaboración intra y extrafamiliar: redes sociales de apoyo y e) preservación de la vida: actividades de crianza y cuidado de los niños, cuidado y atención de la salud, morbilidad y mortalidad. Adicionalmente se analizaron las características de los integrantes de la familia y las condiciones de la vivienda.

La variable dependiente, desnutrición, fue medida a través de un niño índice (entre 6 y 23 meses de edad) identificado en cada familia. De existir dos o más niños dentro del grupo de edad, se denominó como niño índice al que presentara algún grado de desnutrición; en el caso contrario se seleccionó al niño de mayor edad.

Definición de caso. Se consideró caso cuando en la familia hubo un niño índice que presentó algún grado de desnutrición (valor $\mathrm{z}$ del indicador talla para la edad igual o menor a -1.64 (valor de $p=0.05$ considerando una cola) de la mediana de referencia internacional sobre crecimiento del Centro Nacional de Estadísticas de Salud de los Estados Unidos y de la Organización Mundial de la Salud [NCHS/WHO]). ${ }^{23}$

Se consideró control cuando en la familia hubo un niño índice eutrófico de acuerdo con el mismo criterio.

Dos enfermeras capacitadas para la entrevista y estandarizadas para mediciones antropométricas por medio del procedimiento validado por Flores-Huerta y colaboradores, ${ }^{24}$ realizaron visita domiciliaria y entrevista al jefe de familia o responsable del o los niños mediante un cuestionario estructurado para la obtención de las variables; para las mediciones antropométricas (peso y talla) se utilizaron infantómetros de aluminio con precisión milimétrica construidos para el estudio y básculas electrónicas pesa bebé Tanita ${ }^{\mathrm{MR}}$ modelo No. 1583, con precisión de 10 g. Para controlar la autenticidad de la información se incluyeron apartados para el registro de los nombres, fechas de nacimiento de los hijos y el uso de documentos oficiales como la Cartilla
Nacional de Vacunación, la realización de una prueba piloto en un municipio con población similar a la estudiada, así como supervisión y asesoría permanentes.

Se hizo análisis descriptivo por medio de frecuencias simples, absolutas y relativas. A las variables independientes se les hizo análisis bivariado, cálculo de razón de momios cruda (RMc) e intervalos de confianza al 95\% (IC95\%). Se construyeron modelos de regresión logística no condicionada por cada una de las estrategias familiares de vida, así como un modelo global, agregando en forma secuencial las variables que mostraron asociación significativa con desnutrición, y de acuerdo con un esquema conceptual basado en procesos de toma de decisiones, en orden cronológico, este esquema plantea las condiciones sociales de los padres al formar la unidad familiar, su comportamiento reproductivo, la forma en que obtienen sus recursos, cómo se organizan los mismos, las acciones específicas que realizan para la preservación de la vida de sus integrantes y por último la forma como se relacionan con su entorno.

\section{Resultados}

Se visitaron 633 domicilios de las familias que cumplieron con los criterios de elegibilidad, logrando la entrevista en 605 (95.6\%) de ellas; los informantes fueron las madres del menor en $76.5 \%$ de las veces, las unidades familiares fueron constituidas a partir de uniones legales (matrimonio civil o religioso en $77.4 \%$ contra $22.6 \%$ de uniones consensuales (unión libre y padre o madre solteros). Se encontraron 445 (73.6\%) de niños índice que reunieron el criterio de controles, y 160 con desnutrición (26.4\%) que se definieron como casos, de acuerdo con la definición establecida. Por encontrar mayor proporción de casos que lo esperado en el cálculo muestral, se incluyeron a todas las familias; lo anterior permitió obtener un poder de 99.0\%.

Se efectuaron 46 entrevistas dobles, que incluyeron las mediciones antropométricas, para evaluar variabilidad interobservador, y se calcularon los coeficientes de correlación intraclase para las variables numéricas, de concordancia de Kappa y de Kappa ponderada para las variables nominales y ordinales;, ${ }^{25-27}$ la concordancia fue superior a 0.80 y la correlación inferior $+/-0.30$.

El cuadro I muestra las principales características de los niños índice casos y controles. Se encontraron diferencias significativas en el orden de nacimiento, con mayor proporción de primogénitos en los controles, así como mejor escolaridad en ambos padres y mejores condiciones de vivienda (menor frecuencia de hacinamiento y mayor proporción de drenaje). 


\section{Características generales de la población} de estudio.Tlaxcala, México, 1998

\begin{tabular}{|c|c|c|c|c|}
\hline \multirow[b]{2}{*}{ Niño índice } & \multicolumn{2}{|c|}{$\begin{array}{c}\text { Casos } \\
n=160\end{array}$} & \multicolumn{2}{|c|}{$\begin{array}{l}\text { Controles } \\
n=445\end{array}$} \\
\hline & $n$ & $\%$ & $n$ & $\%$ \\
\hline \multicolumn{5}{|l|}{ Sexo } \\
\hline Masculino & 88 & 55.0 & 199 & 44.7 \\
\hline Femenino & 72 & 45.0 & 246 & 55.3 \\
\hline
\end{tabular}

Edad (meses)

\begin{tabular}{cllll}
6 a 11 & 43 & 32.6 & 154 & 34.6 \\
\hline 12 a 17 & 58 & 34.0 & 148 & 33.3 \\
\hline 18 a 23 & 59 & 33.4 & 143 & 32.1
\end{tabular}

Orden de nacimiento

\begin{tabular}{lrrrr} 
Primogénitos & 43 & 26.9 & 177 & 39.8 \\
\hline 20 o posterior & 117 & 73.1 & 268 & 50.2
\end{tabular}

Padre

Escolaridad

\begin{tabular}{lrrrr} 
Menor a 6o grado & 26 & 16.3 & 27 & 6.1 \\
\hline 6 o a 8 & 69 & 43.1 & 143 & 32.1 \\
\hline 9o o más & 65 & 40.6 & 275 & 61.8
\end{tabular}

Madre

Escolaridad

\begin{tabular}{lllrr} 
Menor a 6o grado & 31 & 19.4 & 38 & 8.5 \\
\hline 60 a 8 & 78 & 48.8 & 156 & 35.1 \\
\hline 9o o más & 51 & 31.8 & 251 & 56.4
\end{tabular}

Vivienda

\begin{tabular}{lrrrr} 
Propia & 106 & 66.3 & 296 & 66.5 \\
\hline Compartida & 31 & 19.4 & 100 & 22.5 \\
\hline Prestada/rentada & 23 & 14.3 & 49 & 11.0
\end{tabular}

Personas por recámara

\begin{tabular}{llrrr}
1 a 4 & 93 & 58.1 & 353 & 79.3 \\
\hline 5 o más & 67 & 41.9 & 92 & 20.7 \\
\hline Drenaje & 86 & 53.8 & 320 & 71.9
\end{tabular}

$N=605$

Los resultados del análisis multivariado por estrategia, en el que se incluyeron todas las variables que fueron estadísticamente significativas en el análisis crudo, (cuadros II y III) mostraron que los siguientes factores se encontrarían teóricamente en una familia con un niño desnutrido entre sus integrantes (cuadro IV): a) Formación de la familia. La unidad constituida por mujeres que tenían menos de 20 años de edad al momento del nacimiento de su primogénito, con un intervalo, entre el nacimiento del hermano mayor y el correspondiente al niño índice, menor a 36 meses y que al momento de la visita estuvo integrada por seis o más personas

b) Obtención de recursos. Familia con un ingreso per capita mensual menor a \$385.00 (38 dólares)

c) Organización de recursos. Familia que destinó más de $25.0 \%$ del ingreso mensual para el pago de alimentos y en la cual la madre invirtió menos de 10.5 horas por semana para las actividades de crianza y cuidado de los niños

d) Colaboración intra y extrafamiliar (apoyo social). Familia que no contó con servicios proporcionados por Instituciones de Seguridad Social

e) Preservación de la vida. Familia en la cual el niño índice inició su ablactación después de los seis meses, cuya madre invirtió para su alimentación hasta 25 minutos por cada sesión. Además durante los seis meses previos a la visita no acudieron a un servicio de salud para la vigilancia del estado nutricional del niño

f) Los factores socioeconómicos que mostraron diferencias significativas fueron la baja escolaridad de los padres (seis años o menos), condiciones de vivienda desfavorables representadas por hacinamiento y carencia de servicios públicos como drenaje.

Las variables anteriores, al ser incluidas en el modelo final (cuadro V), generaron el siguiente escenario:

- Las madres de familia tuvieron una baja escolaridad, con una conducta reproductiva caracterizada por el intervalo entre los nacimientos del hermano mayor y el niño índice menor a 36 meses

- En la unidad familiar el ingreso per capita fue menor a $\$ 385.00$ (38 Dls)

- Las madres destinaron menos de 10.5 horas por semana para las actividades relacionadas con la crianza y el cuidado de los niños

- Al momento de la entrevista, cinco o más de sus integrantes compartieron un cuarto para dormir.

\section{Discusión}

Las perspectivas teóricas, sociológicas, y microeconómicas que permiten analizar los determinantes de la salud de los miembros de la familia tienen puntos de convergencia importantes. El concepto aquí propuesto 


\section{Cuadro II \\ VARIABLES SIGNIFICATIVAS EN EL ANÁLISIS BIVARIADO PARA FORMACIÓn DE LA UNIDAd FAMILIAR, POR CADA ESTRATEGIA.TLAXCALA, MéXICO, 1998}

Formación de la

Unidad familiar

Edad de la madre al momento de la unión

Menor de 20 años

Edad de la madre al momento del nacimiento del primer hijo Menor de 20 años

Edad de la madre al momento del nacimiento del hijo subsecuente Menor de 20 años

Intervalo entre nacimientos $1 \stackrel{0}{ }$ y $2 \stackrel{0}{\text {. }}$
$\quad$ Menor a 24 meses

Intervalo entre nacimiento anterior y el correspondiente al niño índice

Menor a 36 meses

$N$ úmero de hijos nacidos vivos

50 más

Uso de métodos de control de la fertilidad

No

$N$ úmero de integrantes por unidad familiar

2 a 5

60 más

Razón adultos/niños

Menor a $0.67(2 / 3)$

O btención de recursos

0 cupación del jefe de familia

O brero, prestador de servicios

Empleado comerciante, Profesional independiente

Ingreso mensual familiar

Hasta dos salarios mínimos

Ingreso per capita mensual

Menor a $\$ 385.00$ (38 D ls.)

$N=605$
Casos Controles RM IC $95 \%$

$n=160 \quad n=445 \quad$ (cruda)

$\begin{array}{llll}63.8 & 51.5 & 1.66 & 1.14-2.41\end{array}$

$\begin{array}{llll}53.1 & 37.8 & 1.87 & 1.30-2.69\end{array}$

$\begin{array}{llll}21.9 & 10.3 & 2.43 & 1.50-3.94\end{array}$

$\begin{array}{llll}23.8 & 14.6 & 1.82 & 1.16-2.85\end{array}$

$\begin{array}{llll}46.9 & 28.5 & 2.21 & 1.52-3.21\end{array}$

$\begin{array}{llll}31.3 & 19.1 & 1.92 & 1.28-2.90\end{array}$

$\begin{array}{llll}51.9 & 40.4 & 1.59 & 1.10-2.28\end{array}$

$\begin{array}{llll}46.3 & 60.2 & 1.76 & 1.22-2.53\end{array}$

$53.7 \quad 39.8$

$\begin{array}{llll}23.8 & 16.2 & 1.61 & 1.04-2.51\end{array}$

$91.9 \quad 81.6$

$2.55 \quad 1.38-4.73$

$\begin{array}{llll}58.7 & 48.5 & 1.51 & 1.05-2.17\end{array}$

$\begin{array}{llll}69.4 & 44.5 & 2.83 & 1.92-4.15\end{array}$ de "estrategias familiares de vida", de connotación sociológica y el concepto de "producción de salud en el hogar, ${ }^{28,29 "}$ de corriente microeconómica, tiene similitudes con respecto a sus componentes, aunque el abordaje de análisis es diferente.

Ambos modelos establecen que la salud y nutrición de los niños (o los integrantes de la familia) dependen de un conjunto de insumos importantes entre los cuales podemos mencionar calidad de la alimentación (que determina el ingreso de nutrientes), cantidad y calidad del cuidado que la familia proporciona, caracterís- ticas individuales (edad y sexo del niño), características de la familia (escolaridad de los padres, ingresos económicos, etcétera) y características del medio ambiente. La combinación de estos factores permite identificar o formular modelos de análisis del estado de salud de los integrantes de la familia, con el supuesto de que éste será diferente para cada uno de ellos. Los más vulnerables son los menores de cinco años y las mujeres

Los resultados del estudio mostraron que existe un proceso de toma de decisiones en las familias y éstas obedecen a diferentes alternativas. Las decisiones en el 


\section{Cuadro III \\ VARIABLES SIGNIFICATIVAS EN EL ANÁLISIS BIVARIADO PARA ORGANIZACION INTRAFAMILIAR, POR CADA ESTRATEGIA. TLAXCALA, MÉXICO, 1998}

O rganización intrafamiliar de los recursos

Porcentaje de ingreso mensual familiar erogado para compra de alimentos Mayor a $25 \%$

Tiempo de trabajo doméstico destinado para la crianza y cuidado de los niños Menor a 10.5 horas por semana

Colaboración intra y extrafamiliar (apoyo social) Seguridad Social

No

Preservación de la vida

Inicio de ablactación

Posterior a los 6 meses de edad

Momento del destete

Posterior a los 15 meses de edad

Duración de sesiones de alimentación Hasta 25 minutos

Demanda de atención de niño sano

Menos de tres consultas en 6 meses previos

Actividades recreativas en grupo

No realizan

$\begin{array}{cccc}\begin{array}{c}\text { Casos } \\ n=160\end{array} & \begin{array}{c}\text { Controles } \\ n=445\end{array} & \begin{array}{c}\text { RM } \\ \text { (cruda) }\end{array} & \begin{array}{c}\text { IC } \\ 95 \%\end{array} \\ 87.5 & 80.0 & 1.75 & 1.04-2.95 \\ 66.9 & 51.2 & 1.92 & 1.32-2.80 \\ 62.5 & 47.2 & 1.86 & 1.29-2.70 \\ 23.1 & 12.8 & 2.05 & 1.29-3.25 \\ 26.3 & 17.3 & 1.70 & 1.11-2.61 \\ 36.9 & 48.1 & 0.63 & 0.43-0.91 \\ 90.0 & & & \\ 12.5 & 60.2 & 2.22 & 1.26-3.91 \\ & & & 1.20-4.07\end{array}$

$N=605$

Cuadro IV

Modelos PRedictivos de desnutricion por CAdA estrategia.Tlaxcala, México, 1998

Formación de la unidad familiar

Intervalo menor a 36 meses entre el nacimiento anterior y el niño índice

Edad de la madre menor a 20 años al momento del nacimiento del primer hijo

Familia con 6 o más integrantes

Obtención de recursos familiares

Ingreso per capita mensual menor a \$ 385.00 (38 D ls.)

O rganización intrafamiliar de los recursos

Menos de 10.5 horas por semana para la crianza y cuidado de los niños

Más de $25.0 \%$ del ingreso mensual familiar gastado en alimentos

Colaboración intra y extrafamiliar.

Carencia de servicios por parte de instituciones de seguridad social $\quad 0.4244 \quad 1.53 \quad 1.04-2.25$

Preservación de la vida

Menos de tres consultas de vigilancia y control de crecimiento y desarrollo, durante los últimos 6 meses

Ablactación después de los 6 meses de edad

Hasta 25 minutos de duración por cada sesión para alimentación al niño índice

Factores socioeconómicos

Hacinamiento

Escolaridad de la madre ( 6 años o menos)

Escolaridad del padre (6 años o menos)

Carencia de drenaje

*Intervalo de confianza, todos los modelos fueron controlados por edad del niño índice

$\mathrm{N}=605$
B Razón de Momios I.C.* 95\%

$\begin{array}{lll}0.7434 & 2.10 & 1.44-3.07\end{array}$

$0.5372 \quad 1.71 \quad 1.18-2.49$

$\begin{array}{lll}0.4835 & 1.62 & 1.12-2.36\end{array}$

$0.9812 \quad 2.66 \quad 1.79-3.97$

$\begin{array}{lll}0.7005 & 2.01 & 1.37-2.95\end{array}$

$\begin{array}{lll}0.6612 & 1.94 & 1.14-3.30\end{array}$

$\begin{array}{lll}0.7337 & 2.08 & 1.18-3.69\end{array}$

\begin{tabular}{lll}
0.6353 & 1.89 & $1.18-3.01$ \\
\hline
\end{tabular}

$\begin{array}{lll}-0.4225 & 0.65 & 0.45-0.95\end{array}$

$\begin{array}{lll}0.6190 & 1.86 & 1.22-2.83\end{array}$

$\begin{array}{lll}0.5673 & 1.76 & 1.22-2.83 \\ 0.4453 & 1.56 & 1.03-2.38\end{array}$

$\begin{array}{lll}0.4453 & 1.56 & 1.03-2.38\end{array}$

$\begin{array}{lll}0.4376 & 1.55 & 1.04-2.317\end{array}$ 


\section{Cuadro V Modelo global predictivo para desnutrición*.Tlaxcala, México, 1998}

\begin{tabular}{|c|c|c|c|}
\hline Variable & $\beta$ & $\begin{array}{c}\text { Razón } \\
\text { de momios }\end{array}$ & $\begin{array}{c}1 \mathrm{C} \\
95 \%\end{array}$ \\
\hline Menos de 10.5 horas semanales destinadas para la crianza y cuidado de los niños & 0.6750 & 1.96 & $1.31-2.93$ \\
\hline Intervalo menor a 36 meses entre el nacimiento anterior y el niño índice & 0.5715 & 1.77 & $1.18-2.66$ \\
\hline Baja escolaridad de la madre (6 años o menos) & 0.5715 & 1.77 & $1.17-2.69$ \\
\hline Hacinamiento (5 o más personas por recámara) & 0.5588 & 1.75 & $1.13-2.71$ \\
\hline $\begin{array}{l}\text { Ingreso per capita menor a \$ } 385.00 \text { (38 Dls.) } \\
\begin{array}{l}* \text { Controlado por edad del niño índice } \\
N=605\end{array}\end{array}$ & 0.5553 & 1.74 & $1.12-2.71$ \\
\hline
\end{tabular}

ámbito individual y familiar están influenciadas por variables socioeconómicas, como educación e ingreso. Las familias con mayor nivel educativo tienen un mejor estado de salud. Las mujeres con mayor educación mantienen mejores condiciones higiénicas y son más receptivas a la información en salud; adicionalmente, son más capaces de transformar los insumos de la familia en salud. ${ }^{30}$ Algunos de los factores de riesgo identificados en este estudio ya han sido destacados en otros trabajos; entre ellos se encuentra el intervalo reducido entre dos nacimientos en la familia, ${ }^{31}$ y el tiempo que destinó la madre de familia a la crianza y cuidado de los niños, este último similar a la relación con la higiene observada en niños en otras áreas de México. ${ }^{32}$ Las características de la ocupación del padre o perceptor económico principal se ha encontrado como uno de los indicadores más sensibles de las condiciones económicas de la familia, y como un fuerte predictor de desnutrición. ${ }^{33}$

El ingreso en la familia tiene una relación directa con el estado de salud. La distribución del ingreso dentro del hogar afecta la salud. A mayor ingreso, la probabilidad de un mejor estado de salud se incrementa para todos los miembros de la familia, mientras que, con un ingreso bajo, comienza un proceso de selección y aparecen diferencias en la distribución de los bienes (incluidos los alimentos) entre los individuos de la familia, lo que genera distribución inequitativa. ${ }^{34}$

La baja utilización de los servicios de salud, identificada como poca asistencia a los programas de atención al niño, se encontró como factor de riesgo, lo que refleja la necesidad de una mayor información a la población acerca de los beneficios de la evaluación del crecimiento y desarrollo del niño para la identificación temprana de condiciones desfavorables.

La búsqueda de atención médica es un fenómeno complejo que obedece, primariamente, a la percepción de la familia con respecto a la enfermedad, y en su capacidad para solventar los gastos que esto representa. ${ }^{35}$ La decisión depende del beneficio inmediato observable; en el caso de un niño enfermo, la familia solicitará atención médica para recuperar su salud. No ocurre la misma situación en programas preventivos o de detección, porque la familia no alcanza, en muchos casos, a percibir el beneficio de las acciones preventivas porque éstas no tienen un efecto inmediato o tangible.

El comportamiento que mostró un resultado inverso al esperado fue la menor duración de las sesiones de alimentación, que resultó protector para desnutrición en los modelos por estrategia, ya que el supuesto teórico para la medición planteó que más tiempo invertido para la alimentación se relaciona con un mejor estado nutricional del niño y puede estar relacionado con las características del cuidador; se ha observado que es posible estimular el interés del niño para su alimentación si se tiene la convicción de que esta actividad representa una de aquellas de mayor prioridad para mantener un adecuado estado nutricional de éste; es posible que la estimulación sólo se dé como cuidado compensatorio cuando las condiciones de nutrición son adversas, lo que explicaría los resultados encontrados. ${ }^{36}$ Debido a las características del estudio, que no permitió una observación directa de las variables relacionadas con los patrones de alimentación, no fue posible discriminar algunas otras condiciones que se han encontrado asociadas con el estado nutricional del niño, como son la cantidad y calidad de los alimentos que se ingieren de acuerdo con el tipo de sesión (desayuno, almuerzo, comida, merienda o cena), y las ventajas o desventajas de contar con cuidadores secundarios o alternos.

El procedimiento utilizado para la construcción de los modelos de análisis pretendió hacer evidentes las diversas estrategias de vida de la familia, sus relaciones con el contexto social y el logro de su sobrevivencia. ${ }^{37}$ 
El modelo global que incluye a todas las estrategias familiares, debe analizarse tomando en consideración que el lugar donde se llevó a cabo el estudio se encuentra en una fase de transición entre una forma de vida tradicional (rural) y la modernización del medio urbano; por tal motivo se observó la coexistencia de características de las estrategias familiares de vida del pasado, relacionadas con la educación y la vida reproductiva de las mujeres. ${ }^{38}$

Es evidente que la interpretación de los resultados con base en este modelo tiene ventajas y limitaciones. La ventaja principal consiste en medir la magnitud de la asociación individual de cada una de las variables del modelo con el resultado de interés. Sin embargo, es necesario aceptar la limitación que aduce el diseño seleccionado para el estudio, pues al hacer una sola medición se pierde la oportunidad de identificar escenarios cambiantes a través del tiempo y, por ende, sustentar las probables explicaciones del proceso en general.

En conclusión, es relevante reconocer la importancia de la familia como productora y responsable de las condiciones de salud (y enfermedad) de sus miembros Los niños, como integrantes más vulnerables, dependen de la familia con respecto a su alimentación y al resto de los bienes que se les debe proporcionar. El análisis de las estrategias de vida de las familias permite estimar su capacidad para interactuar con el entorno y para transformar los insumos en bienes que después son repartidos entre sus integrantes. El enfoque utilizado para la construcción de modelos predictivos permitió la comprensión del papel de algunos factores de riesgo de desnutrición, en niños menores de dos años de edad. El diseño de programas efectivos debe considerar que las estrategias familiares de vida juegan un papel determinante para la distribución de los recursos al interior de la familia, que forman parte del proceso de toma de decisiones para la búsqueda de la sobrevivencia de cada uno de sus integrantes y que se encuentran relacionadas con el cuidado de la salud.

\section{Agradecimientos}

Los autores agradecen la colaboración durante la fase de recolección de la información a las licenciadas en enfermería María Inés Espinosa Aguila y Ana María Pérez Hernández.

\section{Referencias}

1.Schroeder DG, Brown KH. El estado nutricional como factor predictivo de la supervivencia infantil: síntesis de la asociación y cuantificación del impacto glo bal. Bol 0 ficina Sanit Panam 1995; 2:121-134.
2. World Bank. World Development report 1993: Investing in health. W ashington, D.C.: 0 xford University Press, 1993: 77.

3.W orld Bank. Enriching lives: 0 vercoming vitamin and mineral malnutrition in develo ping countries.W ashington D.C.:W orld Bank, 1994: 2.

4. Rivera-D ommarco J, González-C osío T, Flores M, Hernández-Avila M, Lezana MA, Sepúlveda-Amor J. Déficit de talla y emaciación en menores de 5 años en distintas regiones y estados de México. Salud Publica Mex 1995; 37: 95-107.

5. Secretaría de Salud, Instituto N acional de Salud Pública. Instituto N acional de Estadística, Geografía e Informática. Encuesta N acional de Nutrición 1999.Tomo I. Resultados. N iños menores de cinco años. México, D.F.:SSA, IN SP, IN EGI, 2000.

6.Yoom PW, Black RE, Moulton LH, Becker S.The effect of malnutrition on the risk of diarrhoeal and respiratory mortality in children $<2 \mathrm{y}$ of age in Cebu Philippines. Am J N utr 1997; 65: 1070-1077.

7. Bôhler E, Bergstrôm S. Frequent diarrhoeas in early childhood have sustained effects on the height, weight and head circumference of children in East Bhutan. Acta Paediatr 1996; 85: 26-30.

8. Méndez MA, Adair LS. Severity and timing of stunting in the first two years of life affect performance on cognitive tests in late childhood.J N utr 1999; 129: 1555-1562.

9. Ramakrishnan U, Martorell R, Schroeder DG, Flores R. Role of intergenerational effects on linear growth. Symposium: $C$ auses and etiology of stunting. J N utr 1999; 129: 544S-549S.

10. Frongillo Ea Jr. Symposium:C auses and etiology of stunting. Introduction. J Nutr 1999; 129: 529S-530S.

11. Rosado JL, López P,Muñoz E, Martínez H ,Allen HL.Zinc supplementation reduced morbidity, but neither zinc nor iron supplementation affected growth or body composition of Mexican preschoolers. Am J C lin N utr 1997; 65: 13-19.

12. LiuYX, Jalil F, Kalberg J. Risk factors for impaired length growth in early life viewed in terms of the infancy-childhood-puberty (ICPO) growth model. Acta Paediatr 1998; 87: 237-243.

13. Stephensen CB. Burden of infection on growth failure. Symposium: Causes and etiology of stunting.J N utr 1999; 129: 534S-538S.

14. Black MM, Krishnakumar A. Predicting longitudinal growth curves of height and weight using ecological factors for children with and without early growth deficiency. Symposium: C auses and etiology of stunting. J N utr 1999; 129: 539S-543S.

15. Adair LS, Guilkey DK. Age-specific determinants of stunting in filipino children.J N utr 1997; 127: 314-320.

16. Frongillo EA, Jr de $\mathrm{O}$ nis $M, H$ anson $K M$. Socioeconomic and demographic factors are associated with worldwide patterns of stunting and wasting of children.J N utr 1997; 127: 2302-2309.

17.W ishik SM,Van Der Vynckt S.The use of nutritional "positive deviants" to identify approaches for modification of dietary practices. Am J Public Health 1976; 66: 38-42.

18. Torrado S. El enfoque de las estrategias de vida en América Latina. 0 rientaciones teórico metodológicas. Buenos Aires: Centro de Estudios Urbanos y Regionales, 1983; Cuadernos del CEUR, 2.

19. Torrado S. Clases sociales, familia y comportamiento demográfico. 0 rientaciones metodoló gicas. Demografía y Economía 1978; 12: 343-376. 20. Blanes J. D e los valles al Chapare, estrategias familiares de un contexto de cambios. Cochabamba, Bolivia: Centro de estudios la realidad económica y social, 1983; Serie Cochabamba, N 0. 4.

21. De la Rocha GM. O rganización y reproducción de las unidades domésticas de la clase trabajadora en Guadalajara. México, D.F.: C entro de Investigaciones y Estudios Superiores en Antropología Social, 1986.

22. Bronfman N M. Multimortalidad infantil y familia. Dinámica, estructura y riesgo diferenciado. Perinatol Reprod Hum1995; 9: 11-28.

23. D epartment of Health Education and W elfare. Public Health Service, $\mathrm{N}$ ational Center for Health Statistics. N C HS G rowth curves for children. Birth-18 years. United States U.S.Vital and Health Series 11, N umber 165. Hyattsville, (MD): D HEW Publication (PHS), 1977; 78: 1650. 
24. Flores-Huerta S, Villalpando C, Fajardo-Gutiérrez A. Evaluación antropométrica del estado de nutrición en los niños. Procedimientos estandarización y significado. Bol Med Hosp Infant Mex 1990; 47:725-735. 25. Burdock El, Fleiss JL, Hardesty AS. A new view of interobserver agreement. Pers Psychol 1963;16: 373-384.

26. Fajardo-Gutiérrez A, Yamamoto-Kimura LT, Garduño-Espinoza J, Hernández-Hernández D M, Martínez-García MC. Consistencia y validez de una medición en la investigación clínica pediátrica. D efinición, evaluación y su interpretación. Bol Med Hosp Infant Mex 1991; 48: 367-381.

27. Landis RJ, Koch GG. The measurement of observer agreement for categorical data. Biometrics 1977; 33: 159-174.

28. DaVanzo J.G ertler P. Household production of health:A microeconomic perspective on health transitions. A Rand Note, N-3014-RC 1990. The Rand Corporation. Santa Monica, CA, USA, 1990.

29. Pitt M.A nalyzing human resource effects: Health. En:Demery L,Ferroni $M, G$ riffin $C$. Understanding the social effects of policy reform.W ashington, D.C.:The W orld Bank, 1993

30. SchultzTP. Studying the impacto of household economic and community variables on child mortality. Popul Develop Rev 1984;10:215-235.

31. D'Souza RM. Housing and enviromental factors and their effects on the health of children in the slums of Karachi, Pakistán.J Biosoc Sci 1997; 29: $271-281$.
32. Gómez de León CJ, Zúñiga HE, López RA, Rodríguez DC , Raphael ZT. Pobreza, desnutrición y atención a la salud en una zona marginada de la ciudad de México: C himalhuacán. Serie: Estudios Sectoriales. México, D.F.: CONAPO, 1996.

33. Kikafunda JK, W alker AF, Collet D, Tumwine JK. Risk factors for early childhood malnutrition in U ganda. Pediatrics 1998; 102: E45.

34. D eatonA.C ase A.A nalysis of household expenditures. Living Standards measurement study (LSMS) W orking Paper 28.W ashington, D.C .:TheW orld Bank, 1987.

35. Bitran R. McInnes K. The demand for health care in Latin America. Economic development Institute for the World Bank. W orking Paper No. 46.W ashington, D.C.:The W orld Bank. 1993.

36. Engle P,Zeitlin M.Active feeding behavior compensates for low interest in food among young nicaraguan children.J N utr 1996; 1808-1816.

37. Berman P, Kendall C, Bhattacharyya K. The household production of health. Putting people at the center of health improvement. En: Sirageldin I, MosleyW H, Levine R, SchwobelV, Horiuchi K. ed.Towards more efficacy in child survival strategies. Baltimore, (MD): JHU Publications,1989:1-18. 38. Schlaepfer L, Infante C. Patrones de inicio de la vida reproductiva: su relación con la mortalidad infantil y comportamientos reproductivos futuros. En:Avila H, Lartigue T, comp. Sexualidad y reproducción humana en México. México, D.F.: UIA/Plaza y Valdés, 1996:77-94. 